\title{
Giant Mucinous Cystadenoma of the Appendix With Low Grade Dysplasia Presented as Acute Appendicitis: A Case Report and Literature Review
}

\author{
Aleksandr A. Reznichenko
}

\begin{abstract}
Mucinous cystadenoma of the appendix is an unusual neoplasm, which represents one of the histological subtypes of mucocele. Giant appendiceal cystadenomas are extremely rare and most commonly presented with palpable abdominal mass, chronic right lower quadrant (RLQ) pain, or could be asymptomatic. We present a 51-year-old female with clinical picture of acute appendicitis and a giant mucinous cystadenoma of the appendix with low grade dysplasia.
\end{abstract}

Keywords: Mucocele; Mucinous cystadenoma; Appendicitis; Appendectomy; Colectomy; Pseudomixoma peritonei

\section{Introduction}

Mucinous cystadenoma of the appendix is one of the histological types of mucocele and it accounts for $63-84 \%$ of all cases. Clinical picture of these lesions is unspecific, and most commonly they present with palpable abdominal mass, chronic right lower quadrant (RLQ) pain, or could be asymptomatic. Computer tomography (CT) is the gold standard for the diagnosis. Treatment requires surgery. Extent of the operation depends on the dimension and histological type of the lesion.

Giant mucinous cystadenomas are extremely rare. It is not clear, what should be called a "giant cystadenoma". The vast majority of giant appendiceal cystadenomas were $10 \mathrm{~cm}$ or larger. We accounted 18 patients in the literature, who were diagnosed with mucinous cystadenoma of the appendix with the size of the neoplasm exceeding $10 \mathrm{~cm}$.

We analyzed clinical and radiological features of giant mucinous cystadenomas, as well as differences in surgical strategy.

We report an unusual case of a giant mucinous cystadenoma of the appendix in a 51-year-old female, who presented with clinical picture of acute appendicitis.

Manuscript accepted for publication April 15, 2015

Department of Surgery, Adventist Health Medical Center, 470 N. Greenfield Avenue, Ste 37, Hanford, CA 93230, USA. Email: areznik9@yahoo.com

doi: http://dx.doi.org/10.14740/jcs265w

\section{Case Report}

A 51-year-old female patient with history of obesity, diabetes, high blood pressure, and C-section was presented to the ER with $24 \mathrm{~h}$ of severe RLQ abdominal pain, nausea and chills. Her vitals signs were stable. On exam she had tenderness in RLQ without peritoneal signs. Her white blood cell (WBC) count was 15.2. Abdominal CT showed a tubular structure 11.8 $\times 3.9 \mathrm{~cm}$ in right iliac fossa, with thick wall and broad base, connected to the cecum, with adjacent mesenteric stranding (Fig. 1, 2). Diagnosis of appendiceal mucocele was suspected, and patient was admitted to the hospital. After bowel preparation, patient was taken to the OR. Exploratory laparotomy was performed. Large mass approximately $12 \times 4 \mathrm{~cm}$, connected to the cecum, with broad and hard base was found, and right hemicolectomy was performed (Fig. 3). Frozen section during surgery was inconclusive. Final pathology showed mucinous cystadenoma with low grade dysplasia, chronic active inflammation with extensive mucosal ulceration (Fig. 4). Patient had successful and uneventful recovery. There was no symptoms or recurrence 9 months after operation.

\section{Discussion}

Appendiceal mucoceles, first described by Rokitansky, repre-

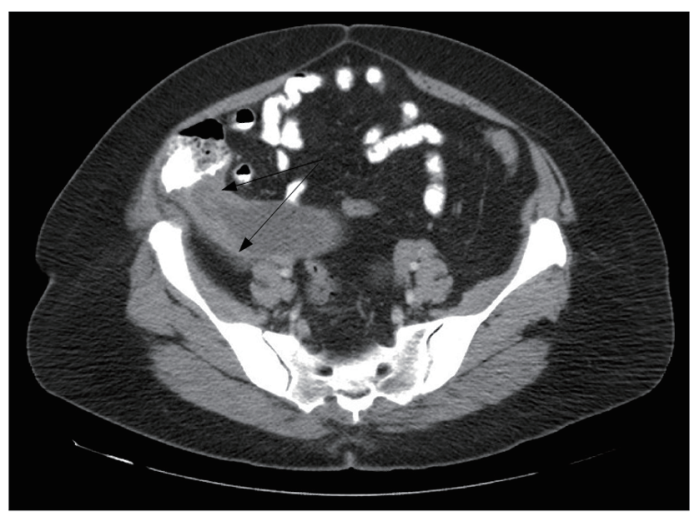

Figure 1. CT abdomen and pelvis with $\mathrm{PO}$ and IV contrast, axial view. Tubular structure in right iliac fossa with broad base and thick wall (arrows). 


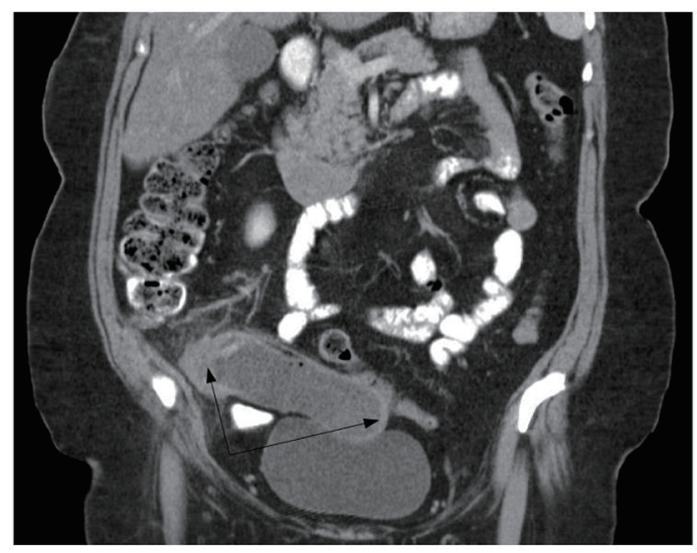

Figure 2. CT abdomen and pelvis with $P O$ and IV contrast, coronal view. Tubular structure in right iliac fossa with broad base and thick wall (arrows).

sent a progressive dilatation of the appendix from intraluminal accumulation of the mucus [1]. They are rare entities with an incidence $0.2-0.7 \%$ in appendectomy (APPY) cases [2-6]. Among all appendiceal tumors, mucocele is second only to carcinoid [7].

Mucoceles of the appendix are classified according to histological characteristics of lumen obstruction. They are divided into simple mucocele or retention cyst, hyperplastic mucocele, mucinous cystadenoma and mucinous adenocarcinoma. The latter two are often referred to as neoplastic mucoceles. Both mucinous cystadenoma and mucinous adenocarcinoma may cause pseudomixoma peritonei, a potentially devastating com-

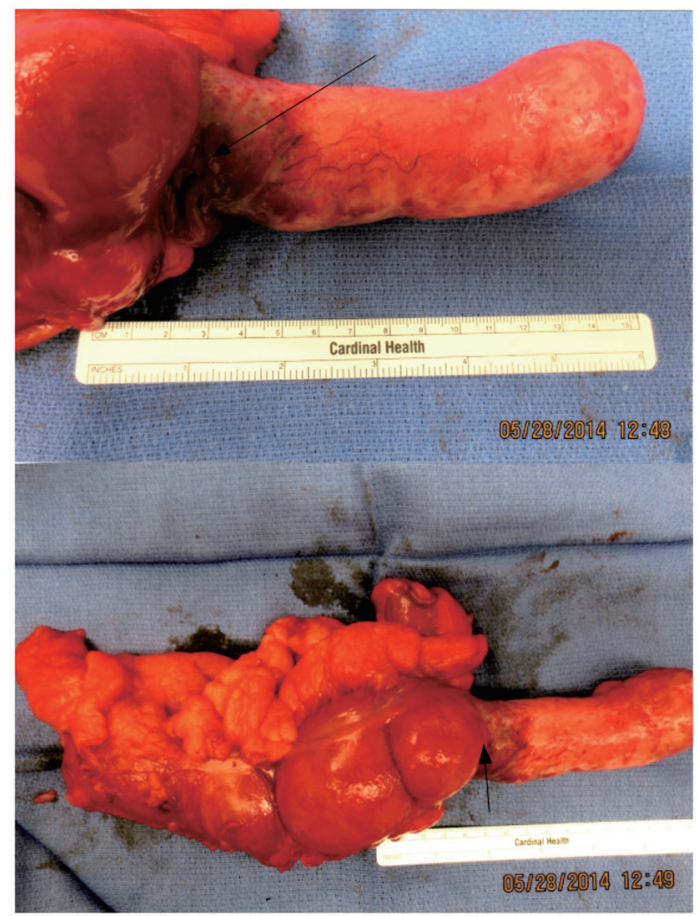

Figure 3. Surgical specimen. Large Appendiceal tumor with thick base and cecal involvement (arrows).

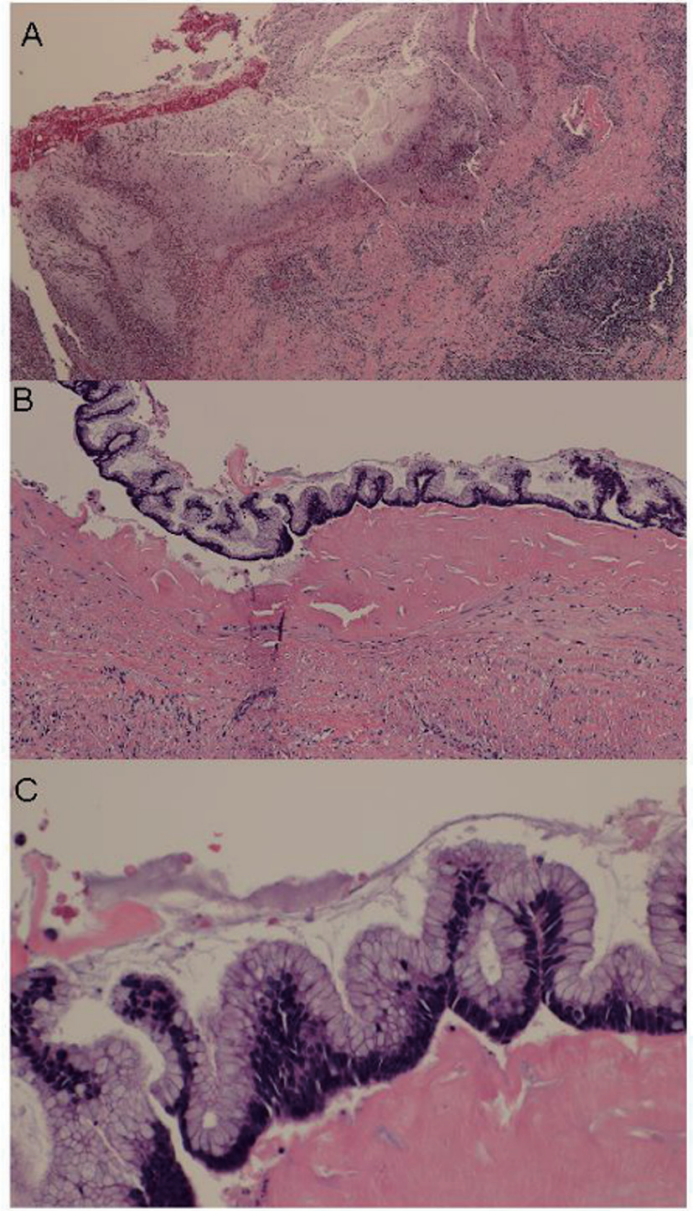

Figure 4. Histological findings. (A) Extensive mucosal ulceration. (B) Cystadenoma with low grade dysplasia. (C) Low grade dysplasia in high power field.

plication of mucocele rupture $[8,9]$.

The size of the non-neoplastic mucoceles (simple and hyperplastic) is smaller than neoplastic mucoceles and generally does not exceed $2 \mathrm{~cm}$; however, there is no difference related to size between benign (cystadenoma) and malignant (adenocarcinoma) neoplastic mucoceles. Hyperplastic mucocele accounts for $5-25 \%$ of cases, mucinous cystadenoma (most common type) for $63-84 \%$, and mucinous cystoadenocarcinoma for $11-20 \%$ of all mucoceles. Both mucinous cystadenoma and cystoadenocarcinoma are characterized by marked distention of the appendiceal lumen up to $6 \mathrm{~cm}[9,10]$.

The clinical presentation of appendiceal mucoceles is usually unspecific; the most common symptom is abdominal pain with or without palpable mass in RLQ $[9,11]$. Very large lesions could be asymptomatic up to $51 \%[12,13]$. Rare complications such as appendicular intussusception have being reported [14].

Preoperative imaging studies help to visualize a cystic mass connected to the cecum. It is generally accepted that CT is better than ultrasound (US) and magnetic resonance imaging (MRI) to diagnose mucocele: however, it is difficult to distinguish between benign and malignant neoplastic mucoceles [9, 15-17]. 


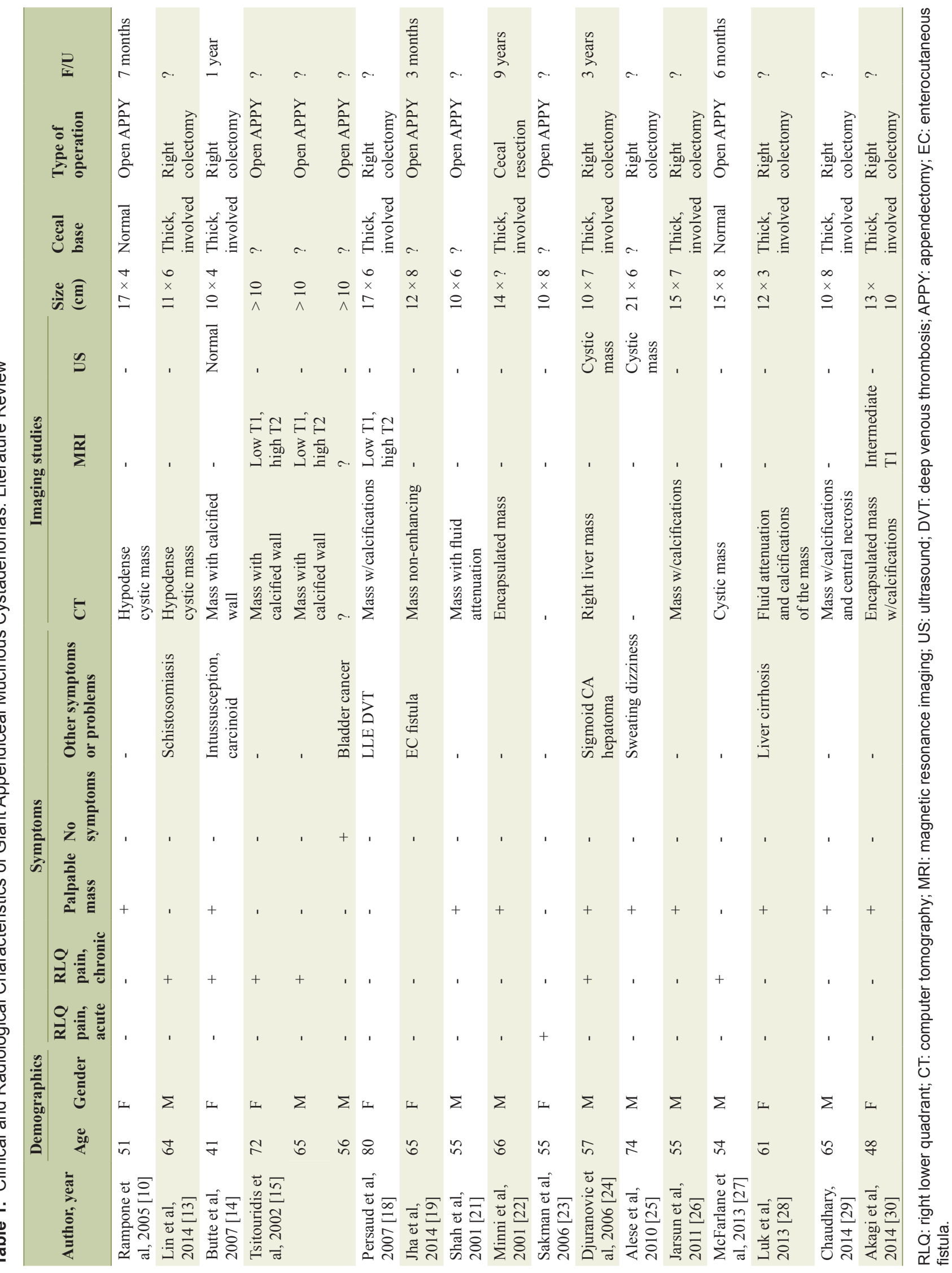


Appendiceal mucocele requires surgical resection. The extent of surgery remains controversial and depends on the size and histology type. For simple and hyperplastic mucoceles of smaller size, APPY is adequate. Cecal resection is indicated for cystadenomas with large base, and hemicolectomy is recommended for cystadenocarcinomas $[9,10,18,19]$. There is no consensus regarding frozen section of the removed specimen during surgery as it is not always accurate [20].

Giant mucinous cystadenomas of the appendix are extremely rare and only few cases have been reported. There is no clear definition of giant cystadenoma. According to the literature, the vast majority of giant appendiceal cystadenomas were described to be bigger than $10 \mathrm{~cm}$ in size $[10,13-15$, 18-30]. We included 18 patients into our literature review, who were diagnosed with mucinous cystadenoma of the appendix with the size of the neoplasm exceeding $10 \mathrm{~cm}$. Patients with cystoadenocarcinoma were not included. Patients with mucocele $10 \mathrm{~cm}$ and larger but without elements of mucinous cystadenoma were not included. We analyzed clinical and radiological characteristics of giant mucinous cystadenoma, as well as different surgical approaches. Results of this analysis are summarized in Table 1.

Giant mucinous cystadenoma of the appendix was seen slightly more often in men, but it was not statistically significant. Majority of the patients were older than 50 years $(89 \%)$. The most common symptom was palpable mass (55\%), followed by chronic RLQ pain (33\%). Absence of symptoms was noticed in one patient $(5 \%)$. Acute onset of RLQ pain was present in the only one patient (5\%), who was emergently taken to the OR and the diagnosis of appendiceal tumor was made intraoperatively [23]. We present another case of giant mucinous cystadenoma of the appendix with the clinical presentation of acute appendicitis. Our case is noticeable because, despite an unusual clinical presentation, the diagnosis of mucocele was suspected prior to surgery and patient was well prepared for the operation.

Imaging studies were important in evaluation of appendiceal neoplasms. CT was more informative among other tests (MRI and US) and most commonly was showing large hypodense encapsulated mass with fluid attenuation and calcified wall.

Decision about extent of surgical resection was made most of the time during surgery based on the thickness and involvement of the cecal base in the pathological process. Laparoscopic approach was not utilized, because of risk of mucocele rupture. Open APPY was considered appropriate for mucinous cystadenomas without involvement of the cecum. Cecectomy or right hemicolectomy were performed in patients with thick appendiceal base and involvement of the cecum, with right hemicolectomy being the preferred approach. We agree with this choice, particularly when frozen section is not really helpful, like it was in our case.

\section{Conclusion}

Giant mucinous cystadenomas of the appendix are extremely rare. They most commonly present with palpable mass, but not in acute settings. We present an unusual case of giant muci- nous cystadenoma, with clinical picture of acute appendicitis. We proposed to define a giant mucinous cystadenoma as an appendiceal neoplasm exceeding $10 \mathrm{~cm}$ in size. CT remains the gold standard for the diagnosis. Extent of surgical resection depends on the histology type, as well as thickness and involvement of the cecal base.

\section{Abbreviations}

RLQ: right lower quadrant; CT: computer tomography; MRI: magnetic resonance imaging; US: ultrasound; DVT: deep venous thrombosis; APPY: appendectomy; EC: enterocutaneous fistula; WBC: white blood cell count; PO: per os; IV: intravenous

\section{References}

1. Rokitansky CF. A manual of pathologica anatomy. Vol 2. English translation of the Vienna edition (1942). Philadelphia: Blancard and Lea, $1855 ; 89$.

2. Zagrodnik DF, Rose DM. Mucinous cystadenoma of the appendix. Can J Surg. 2009;52(2):158-170.

3. Demetrashvili Z, Chkhaidze M, Khutsishvili K, Topchishvili G, Javakhishvili T, Pipia I, Qerqadze V. Mucocele of the appendix: case report and review of literature. Int Surg. 2012;97(3):266-269.

4. Zanati SA, Martin JA, Baker JP, Streutker CJ, Marcon NE. Colonoscopic diagnosis of mucocele of the appendix. Gastrointest Endosc. 2005;62(3):452-456.

5. Woodruff R, McDonald JR. Benign and malignant cystic tumors of the appendix. Surg Gynecol Obstet. 1940;71:750-755.

6. Aho AJ, Heinonen R, Lauren P. Benign and malignant mucocele of the appendix. Histological types and prognosis. Acta Chir Scand. 1973;139(4):392-400.

7. Deans GT, Spence RA. Neoplastic lesions of the appendix. Br J Surg. 1995;82(3):299-306.

8. Higa E, Rosai J, Pizzimbono CA, Wise L. Mucosal hyperplasia, mucinous cystadenoma, and mucinous cystadenocarcinoma of the appendix. A re-evaluation of appendiceal "mucocele". Cancer. 1973;32(6):1525-1541.

9. Jandui Gomes De Abreu Filho, Erivaldo Fernandes De Lira. Mucocele of the appendix: appendectomy or colectomy. J Coloproctol. 2011;31(3).

10. Rampone B, Roviello F, Marrelli D, Pinto E. Giant appendiceal mucocele: report of a case and brief review. World J Gastroenterol. 2005;11(30):4761-4763.

11. Stocchi L, Wolff BG, Larson DR, Harrington JR. Surgical treatment of appendiceal mucocele. Arch Surg. 2003;138(6):585-589; discussion 589-590.

12. Dhage-Ivatury S, Sugarbaker PH. Update on the surgical approach to mucocele of the appendix. J Am Coll Surg. 2006;202(4):680-684.

13. Lin C, Li X, Guo Y, Hu G, Zhang Y, Yang K, Gan Y, et al. Simultaneous giant mucinous cystadenoma of the appendix and intestinal schistosomiasis: 'case report and brief review'. World J Surg Oncol. 2014;12(385. 
14. Butte JM, Torres J, Henriquez IM, Pinedo G. Appendicular mucosal intussusception into the cecum secondary to an intramural mucinous cystoadenoma of the appendix. J Am Coll Surg. 2007;204(3):510.

15. Tsitouridis I, Kouklakis G, Kalambakas A, Papastergiou C, Emmanouilidou M, Goutsaridou F, Tsantiridis C. Giant appendiceal mucoceles of cystadenomas type: CT and MRI evaluation. Report of three cases and review of literature. Ann of Gastroenterology. 2002;15(1):53-57.

16. Vagholkar K, Jain U, Mahadik A, Iyengar M. Mucocele of the appendix. JMSCR. 2014;2(12):3163-3170.

17. Wang H, Chen YQ, Wei R, Wang QB, Song B, Wang CY, Zhang B. Appendiceal mucocele: A diagnostic dilemma in differentiating malignant from benign lesions with CT. AJR Am J Roentgenol. 2013;201(4):W590-595.

18. Persaud T, Swan N, Torreggiani WC. Giant mucinous cystadenoma of the appendix. Radiographics. 2007;27(2):553557.

19. Jha NK, Sinha DK, Anand A, Rai MK, Gandhi A, Yadav J, Yadav SK. Mucinous cystadenoma of the appendix with enterocutaneous fistula: a therapeutic dilemma. Gastroenterol Rep (Oxf). 2015;3(1):86-89.

20. Harris SH, Khan R, Ansari MM, Maheshwari V. Incidentally discovered giant mucocele of the appendix. J Coll Physicians Surg Pak. 2014;24 Suppl 3(S196-197.

21. Shah I, Gupta R, Sanjeev N, Gupta CL. A rare presentation of cystadenoma of appendix as giant retroperitoneal mass. Practitioner. 2001;8(4):250-251.

22. Minni F, Petrella M, Morganti A, Santini D, Marrano D. Giant mucocele of the appendix: report of a case. Dis Co- lon Rectum. 2001;44(7):1034-1036.

23. Sakman G, Parsak C, Akcam T, Alabaz O. Four giant mucoceles of the appendix vermiformis. The Internet $\mathrm{J}$ of Surgery. 2006;9(1).

24. Djuranovic SP, Spuran MM, Kovacevic NV, Ugljesic MB, Kecmanovic DM, Micev MT. Mucinous cystadenoma of the appendix associated with adenocarcinoma of the sigmoid colon and hepatocellular carcinoma of the liver: report of a case. World J Gastroenterol. 2006;12(12):19751977.

25. Alese OB, Irabor DO. Mucinous cystadenoma of the appendix: a case report. Afr Health Sci. 2010;10(1):99-100.

26. Gaby Adriana Alarcon Jarsun, Ariel Shuchleib Cung, Leon Ylgovsky Weintraub, Alvaro Padilla Rodriguez, Alberto Chousleb Kalach, Samuel Shuchleib Chaba An Med (Mex), 2011;56(4):210-217.

27. McFarlane ME, Plummer JM, Bonadie K. Mucinous cystadenoma of the appendix presenting with an elevated carcinoembryonic antigen (CEA): Report of two cases and review of the literature. Int J Surg Case Rep. 2013;4(10):886-888.

28. Luk YS, Lam LRS, Shum JSF, Khoo JLS. Mucinous cystadenoma of the appendix: a rare cause of right lower abdominal mass. Hong Kong J Radiol. 2013;16:e31-34.

29. Chaudhary P. Mucinous cystadenoma of the appendix: A diagnostic dilemma. J of Surgical Academia. 2014;4(1):60-62.

30. Akagi I, Yokoi K, Shimanuki K, Satake S, Takeda K, Shimizu T, Kondo R, et al. Giant appendiceal mucocele: report of a case. J Nippon Med Sch. 2014;81(2):110-113. 